\title{
Time Geography and Wildlife Home Range Delineation
}

JED A. LONG, Spatial Pattern Analysis and Research Lab, Department of Geography, University of Victoria, PO Box 3060 STN CSC, Victoria, BC, Canada, V8W 3R4

TRISALYN A. NELSON, Spatial Pattern Analysis and Research Lab, Department of Geography, University of Victoria, PO Box 3060 STN CSC, Victoria, BC, Canada, V8W 3R4

\section{Pre-print of published version.}

\section{Reference:}

Long, JA, and TA Nelson. 2012. Time Geography and Wildlife Home Range Delineation. Journal of Wildlife Management. 76(2). 407-413.

\section{DOI:}

http://dx.doi.org/10.1002/jwmg.259

\section{Disclaimer:}

The PDF document is a copy of the final version of this manuscript that was subsequently accepted by the journal for publication. The paper has been through peer review, but it has not been subject to any additional copy-editing or journal specific formatting (so will look different from the final version of record, which may be accessed following the DOI above depending on your access situation). 
2 ABSTRACT We introduce a new technique for delineating animal home ranges that is

3 relatively simple and intuitive: the potential path area (PPA) home range. PPA home

4 ranges are based on existing theory from time geography, where an animal's movement is

5 constrained by known locations in space-time (i.e., $n$ telemetry points) and a measure of

6 mobility (e.g., maximum velocity). Using the formulation we provide, PPA home ranges

7 can be easily implemented in a geographic information system (GIS). The advantage of

8 the PPA home range is the explicit consideration of temporal limitations on animal

9 movement. In discussion, we identify the PPA home range as a stand-alone measure of

10 animal home range or as a way to augment existing home range techniques. Future

11 developments are highlighted in the context of the usefulness of time geography for

12 wildlife movement analysis. To facilitate the adoption of this technique we provide a tool

13 for implementing this method.

14 KEY WORDS home range, time geography, potential path area, wildlife movement,

15 GIS, error

\section{INTRODUCTION}

18 Animal home ranges are used to study many aspects of wildlife ecology including habitat

19 selection (Aebischer et al. 1993), territorial overlap (Righton and Mills 2006), and

20 movement impacts of offspring status (Smulders 2009). Home ranges often serve as the

21 primary spatial unit for wildlife research and represent the area to which an animal

22 confines it's normal movement (Burt 1943). Wildlife telemetry data, typically collected

23 with radio or GPS collars, provide a collection of space-time locations for an animal. 
24 Telemetry data are commonly converted to home ranges to identify spatial patterns in

25 animal movement and answer specific research questions.

26 In order to derive animal home ranges, wildlife scientists have used existing

27 methods in geometric topology and spatial smoothing to transform a set of telemetry

28 points into a polygon animal home range. The two most common methods for computing

29 animal home ranges are the minimum convex polygon (MCP), and kernel density

30 estimation (KDE) (Laver and Kelly 2008). MCP continues to be used extensively in

31 wildlife movement analysis (Laver and Kelly 2008) despite considerable drawbacks, such

32 as sensitivity to sampling intensity and outliers, convex assumption, and inclusion of

33 large, unused interior areas (Worton 1987, Powell 2000, Borger et al. 2006). The

34 prevalence of MCP is likely due to its ease of implementation in common GIS platforms

35 and that it requires no input parameters. Kernel density estimation (KDE) has been

36 influential in home range analysis since its introduction by Worton (1989). KDE remains

37 contentious in animal movement analysis due to issues with selecting an appropriate

38 kernel bandwidth (Hemson et al. 2005, Kie et al. 2010), which can significantly impact

39 results (Worton 1989). Unfortunately, KDE based home ranges can be misleading when

40 telemetry points are irregularly shaped (Downs and Horner 2008) or when animals

41 habituate patchy environments (Mitchell and Powell 2008). A number of other lesser

42 used methods also exist (e.g., harmonic mean, Dixon and Chapman 1980, local nearest-

43 neighbor convex hull, Getz and Wilmers 2004, Brownian bridge, Horne et al. 2007,

44 characteristic hull, Downs and Horner 2009), but have yet to become widely adopted.

45 The objective of this article is to demonstrate a new approach for integrating time

46 attributes accompanying telemetry data when calculating animal home ranges. Drawing 
4 | Long and Nelson

47 on concepts from time geography (Hagerstrand 1970), we develop a new approach for

48 computing animal home ranges that explicitly considers the temporal constraints of

49 animal movement. Time is largely ignored in existing home range techniques, and used

50 primarily for separating data into temporal groups such as seasons (Nielson et al. 2003).

51 The value of this method is discussed in context of existing home range research,

52 including existing examples moving towards a time geographic approach.

\section{METHODS}

\section{Background: Time Geography}

55 Time determines bounds on an objects movement in space (Parkes and Thrift 1975). With

56 time geography (Hagerstrand 1970), these constraints are represented as volumes

57 containing all accessible locations in a three dimensional space-time continuum

58 consisting of geographic coordinates $x$ and $y$ and time ( $t$ ) (frequently termed the space-

59 time cube, Kraak 2003, or space-time aquarium, Kwan and Lee 2004). If both starting

60 and end points are known (as with a collection of telemetry fixes) then the space-time

61 prism represents the set of all accessible locations to the object during that movement

62 segment (Figure 1). The projection of the space-time prism onto the geographic plane is

63 termed the potential path area (PPA), and represents all locations accessible to an object

64 given its start and end points and assumed maximum rate of travel (Figure 1). An object's

65 maximum traveling velocity impacts the extent of these volumes into geographic space.

66 < approximate location Figure $1>$

67 Potential Path Area (PPA): A New Measure of Animal Home Range

68 This work will focus on potential uses of PPA in wildlife movement analysis, specifically

69 the calculation of a PPA animal home range. The PPA represents the set of all accessible 
5 | Long and Nelson

70 locations between two known locations in space and time (Miller 2005). Geometrically,

71 the PPA is an ellipse with focal points located at two known locations, the origin and

72 destination. The spatial extent of the PPA depends on the animal's maximum velocity

$73\left(v_{\max }\right)$ which may be explicitly known or empirically estimated from the data.

74 Visually, conceptualizing the creation of a PPA ellipse is best done using the

75 'pins-and-string' method (Figure 2a). Consider placing pins at the known start $(i)$ and end

76 (j) locations of an animal movement segment. A single string is then tied to each point,

77 connecting the two pins. The length of the string is $D_{\max }$, representing the maximum

78 distance the animal can travel given its maximum velocity $\left(v_{\max }\right)$ and the time difference

79 between points $i$ and $j(\Delta t)$.

$80 \quad D_{\text {max }}=v_{\max } \times \Delta t \quad[1]$

81 The PPA ellipse is drawn by moving a pencil around the two points, but inside of the

82 string, keeping the string tight at all times. Any point located along or within the PPA

83 ellipse is reachable by the animal during this movement segment.

84 < approximate location Figure $2>$

85 Mathematically, given that in unconstrained space PPA is an ordinary ellipse, we

86 can derive PPA using parameters of an ellipse related to animal movement in time and

87 space. We define $v_{\max }$ and $\Delta t$ as above, the maximum velocity of the animal and the time

88 difference between known telemetry locations $i$ and $j$. A PPA ellipse is defined using four

89 parameters: a center point, a major axis, a minor axis, and a rotation angle (Figure $2 \mathrm{~b}$ ).

90 The center point is calculated as the midway point between the spatial $(x, y)$ coordinates

91 of telemetry points $i$ and $j$. The major axis $(a)$ is defined as:

$\begin{aligned} a 2 & =D_{\max } \\ & =v_{\max } \times \Delta t\end{aligned}$ 
6 | Long and Nelson

93 With this we can define the minor axis $(b)$ as:

$94 \quad b=\sqrt{a^{2}-d^{2}} \quad[3]$

95 Where $d$ is the Euclidean distance between points $i$ and $j$. Rotation angle $\left(R_{\theta}\right)$ is the angle

96 the ellipse is rotated from the horizontal, and defined using $x$ and $y$ coordinates of

97 telemetry points $i$ and $j$ :

$98 \quad R_{\theta}=\tan ^{-1}\left(\frac{y_{j}-y_{i}}{x_{j}-x_{i}}\right)$

99 Using these parameters we can generate the PPA ellipse for any pair of known locations

100 in space-time.

101 A PPA home range can be computed by generating PPA ellipses for a set of

102 animal locations. A telemetry dataset of $n$ recordings requires calculation of $n$-1 PPA

103 ellipses which are combined to produce the PPA home range (Figure 2c). Formally this is

104 defined as the union of $n-1$ PPA ellipses such that:

$\left.105 \mathrm{PPA}_{\text {HR }}=\mathrm{UPPA}_{i, i+1}\right], \quad i$ in $\{1, \ldots, n-1\}$

106 The mathematical formulation of this method (represented by equations [1] through [5])

107 is easily implemented in a GIS.

108 Estimating $\boldsymbol{v}_{\max }$

109 The PPA home range method requires a single input parameter $v_{\max }$ that has

110 obvious biological connotations and in some cases may be explicitly known based on a

111 fine understanding of an organism's mobility. This parameter could be related to an

112 organism's maximum velocity. For example, cheetahs have a maximum speed of up to

$113120 \mathrm{~km} / \mathrm{h}$ (Sharp 1997); however it is unreasonable to expect a cheetah to maintain that

114 speed over longer intervals, characteristic of telemetry datasets. It is more useful to 
7 | Long and Nelson

115 compute the maximum distance a cheetah could cover in 30 minutes and derive $v_{\max }$ from

116 this. In practice, $v_{\max }$ should relate biologically to the temporal frequency of recordings.

117 In many cases however, a biologically reasonable estimate of $v_{\max }$ will not be

118 explicitly known and a researcher will be required to estimate it from the data. For each

119 pair of consecutive relocation fixes we can compute the segment velocity $\left(v_{i}\right)$ by:

$120 \quad v_{i}=\frac{d_{i}}{t_{i}} \quad[6]$

121 where $d_{i}$ is the distance and $t_{i}$ the time difference between consecutive fixes. Computing

$122 v_{i}$ for all $n-1$ segments will provide a distribution of $v$ values which can be used to

123 generate estimates for $v_{\max }$. The simplest would be to take $\max \left(v_{i}\right)$ - the maximum

124 observed velocity as $v_{\max }$, however this is problematic as it produces a straight-line

125 (degenerative ellipse) between any consecutive pair of fixes that have this maximum

126 value. A more robust approach is to estimate a value for $v_{\max }$ based on the ordered

127 distribution of the $v_{i}$. Following Robson and Whitlock (1964) an estimate of $v_{\max }$ could

128 take the form:

$129 \hat{v}_{\max }=v_{m}+\left(v_{m}-v_{m-1}\right) \quad$ [7]

130 where $v_{i}$ are in ascending order such that $v_{1}<v_{2}<\ldots<v_{m-1}<v_{m}$ and $m=n-1$. This

131 estimate for $v_{\max }$ has an approximate $100(1-\alpha) \%$ upper confidence limit given by:

$132 \mathrm{U}_{\mathrm{Lim}}\left(v_{\max }\right)=v_{m}+\frac{(1-\alpha)\left(v_{m}-v_{m-1}\right)}{\alpha} \quad[8]$

133 Cooke (1979) and van der Watt (1980) have extended the work of Robson and Whitlock

134 (1964) deriving estimates with lower mean squared errors and smaller confidence

135 intervals, at the cost of added complexity. In the case where $v_{m}=v_{m-1}$, the result from [7]

136 will equal $\max \left(v_{i}\right)$ and cause degenerate ellipses to be produced for pairs of consecutive 
137 points that have this maximum value. The method of van der Watt (1980) is

138 advantageous as it avoids the problem of degenerate ellipses through careful selection of

139 the parameter $k$ in the equation:

$140 \quad \hat{v}_{\max }=\left(\frac{k+2}{k+1}\right) v_{m}-\left(\frac{1}{k+1}\right) v_{m-k}$ [9]

141 where $1<k<m$ representing the $k^{\text {th }}$ ordered value of $v_{i}$. This estimate for $v_{\max }$ has an

142 approximate $100(1-\alpha) \%$ upper confidence limit given by:

$\mathrm{U}_{\mathrm{Lim}}\left(v_{\text {max }}\right)=v_{m}+\left(\frac{1}{1 /\left(1-\alpha^{1 / k}\right)-1}\right)\left(v_{m}-v_{m-k}\right)[10]$

144 In the previously stated problem scenario where $v_{m}=v_{m-1}$ it would be useful to take $k$ to

145 be the largest value such that $v_{m-k}<v_{m}$. In general [9] has been shown to be an improved

146 estimator of $v_{\max }$ over [7] (van der Watt 1980), however it requires that the researcher

147 select an appropriate value for $k$. Alternatively, a more conservative analysis could use

148 the upper confidence interval limits (e.g., [8] or [10]) as an estimator for $v_{\max }$.

149 RESULTS

150 For demonstration, we simulate an animal trajectory using a correlated random walk $(n=$

151 2000). Using this data as a surrogate for animal movement data, we calculate animal

152 home range using two common, existing techniques (MCP and KDE) and the new PPA

153 home range approach (Figure 3 a-c). We used the Robson and Whitlock (1964) method

154 given by [7] for estimating the $v_{\max }$ parameter from the data. The temporal sampling

155 interval of telemetry fixes is known to influence output home range size and shape using

156 MCP (Borger et al. 2006) and KDE (Downs and Horner 2008), but also will influence the

157 PPA home range. To demonstrate this effect, we re-sampled our simulated animal 
9 | Long and Nelson

158 trajectory using only $1 / 4(n=500)$ of the points and re-estimated the $v_{\max }$ parameter using

159 [7] (Figure $3 \mathrm{~d}-\mathrm{f})$.

160 < approximate location Figure 3 >

\section{DISCUSSION}

162 In this example, the effect of changing sampling frequency had minimal effect on home

163 range computed using MCP (figure 3 a \& d), however this will not always be the case

164 (Borger et al. 2006). With KDE, fewer points lead to increased uncertainty in the

165 bandwidth selection process, resulting in a wider bandwidth selection, and in general a

166 larger output home range. With the PPA home range method uncertainty is a function of

167 the time between consecutive known locations, rather than the number of points. As a

168 result, PPA home ranges are comprised of fewer, larger ellipses to account for

169 uncertainty in animal location between consecutive known points, and produce larger

170 home range estimates. We suggest that PPA home ranges be employed only when

171 telemetry data are collected using a relatively short sampling interval (e.g., dense GPS

172 telemetry data). In these situations uncertainty between consecutive fixes will be

173 relatively low. In cases where the temporal duration between fixes is substantially longer

174 (e.g., with most VHF collars), the ellipses produced by the PPA algorithm will be large,

175 resulting in significant overestimations of home range size. We withhold from specifying

176 an absolute threshold on sparse telemetry data where the PPA method should not be used

177 as it will be dependant on both the species (e.g., large vs. small mammal) and application

178 (seasonal home range vs. migratory behavior). Comparison of the PPA home range with

179 existing methods (e.g., KDE and MCP) should provide information as to whether or not 
10 | Long and Nelson

180 the PPA approach is appropriate with a given dataset (see Figure 4 and the accompanying

181 discussion below).

182 The conceptual and computational simplicity of the PPA home range may be its

183 greatest asset. The PPA home range can be defined simply as: given a set of sampled

184 locations (telemetry points) the PPA home range contains all locations in geographic

185 space that the animal could have visited. PPA can be easily implemented in a GIS and

186 requires only one input parameter, maximum travelling velocity $-v_{\max }$, that can be

187 derived using biological knowledge or estimated directly from the data (e.g., using [7] or

188 [9]). If telemetry data are categorized into distinct behavioral segments (e.g., Jonsen et al.

189 2005, Gurarie et al. 2009) where differing $v_{\max }$ would be expected, PPA home range

190 analysis could be further enhanced.

191 It is interesting that given its intuitive structure, ideas from time geography are

192 largely absent from wildlife movement research. Baer \& Butler (2000) use time

193 geographic theory for modeling wildlife movement building upon Hagerstrand's (1970)

194 concept of 'bundling', representing animals congregating in space-time. Regions where

195 'bundling' occurs can be used to identify specific ecological activity in groups of animals

196 (e.g., locating scarce resources). Wentz et al. (2003) implement time geographic

197 constraints for animal movement, interpolating between sampled telemetry locations to

198 model movement paths. Time geography volumes are used by Wentz et al. (2003) to

199 constrain random walks between sampled locations. More recently, Downs (2010)

200 presents a novel approach for incorporating time geographic principles, specifically the

201 potential path area (termed geo-ellipse), into kernel density estimation. Downs (2010)

202 uses the geo-ellipse in place of a circular kernel in the density estimation. Several 
203 advantages of this approach are identified, such as replacing subjective selection of

204 kernel bandwidth by an objective parameter - maximum travelling velocity. Time

205 geographic kernel density estimation assigns zero density to regions outside of the PPA

206 home range, creating a utilization distribution density allocated only to accessible

207 regions.

208 Wildlife do not use the space within their home range evenly motivating use of an

209 intensity surface - termed utilization distribution, to analyze animal space use (Jennrich

210 and Turner 1969). Utilization distributions more adequately portray patterns of space use

211 within wildlife home ranges and provide more reliable estimates of overlap and/or

212 fidelity compared with discrete home range methods (Fieberg and Kochanny 2005).

213 However, these advantages come at the cost of added complexity in deriving the

214 utilization distribution with many researchers continuing to use discrete measures of

215 home range over utilization distributions in analysis due to their simplicity (Laver and

216 Kelly 2008). KDE remains the most popular method for computing utilization

217 distributions despite considerable drawbacks with newer (temporally dense) telemetry

218 data (Hemson et al. 2005, Kie et al. 2010). Horne et al. (2007) propose the Brownian

219 bridge approach for computing the utilization distribution. A Brownian bridge is simply

220 defined as the probability a random walk passes through a location given the known start

221 and end points. Like the PPA home range, with the Brownian bridge approach telemetry

222 data are analyzed using pairs of consecutive telemetry fixes. This method relies on a

223 variance parameter $-\sigma_{m}$ that is difficult to interpret but can be estimated from the data

224 using an optimization algorithm. The PPA method is essentially the discrete equivalent of

225 the Brownian bridge approach, but with simple, intuitive, and easy to estimate parameters 
226 that can be straightforwardly computed in a GIS. Getz and Wilmers (2004) propose the

227 use of overlapping local convex hulls to generate a utilization distribution. A similar

228 approach could be adopted with PPA ellipses to generate a utilization distribution based

229 on the areas under overlapping ellipses. The derivation of an overlap-based utilization

230 distribution for PPA ellipses remains an area for future investigation.

231 Wildlife researchers now routinely collect temporally dense telemetry data using

232 sophisticated tracking technologies (e.g., GPS, Tomkiewicz et al. 2010). Such temporally

233 dense telemetry data provide a more detailed and informative view of animal movement.

234 Given continued advancements in technology in the future it is likely that we will be

235 analyzing (near) continuous animal trajectories. This improved representation of animal

236 movement necessarily results in highly autocorrelated movement data. Much attention

237 has been given to the problems autocorrelated telemetry data pose with traditional

238 methods for studying wildlife movement (Swihart and Slade 1985, Otis and White 1999,

239 Fieberg et al. 2010). Many existing methods, developed for use with temporally sparse

240 telemetry data, are ill equipped for dense telemetry data. The PPA home range method is

241 advantageous with temporally dense telemetry data, as it is capable of including rich

242 temporal information into the derivation of home range. With few exceptions (e.g., Horne

243 et al. 2007) existing home range techniques ignore rich temporal information contained in

244 telemetry datasets. Including temporal information in analysis is beneficial as points are

245 no longer considered independent observations, but rather as a sequence of recordings

246 taken over a time period.

247 Certain land cover types (e.g., dense forest, Rempel et al. 1995) can interfere with

248 locating technologies resulting in missing recordings. Missing data points are problematic 
249 in subsequent analysis as bias towards specific cover types can occur (Frair et al. 2004).

250 By explicitly considering the temporal sequencing of points, PPA home ranges adjust for

251 missing telemetry recordings by way of a larger $\Delta t$ value in these areas, providing an

252 unbiased estimator of home range.

253 Commission errors (locations included in the home range but never visited) and

254 omission errors (locations visited but not included in the home range) are important

255 properties of output home range polygons that require careful consideration (Sanderson

256 1966). All home range methods short of a direct trace of an animal's movement path will

257 include commission errors. Omission errors occur with most methods, but can be avoided

258 by substantially overestimating home range size. This is equivalent to selecting an overly

259 large bandwidth with KDE. Substantial overestimation limits utility for wildlife research

260 as the signature of animal behavior is masked. The PPA home range method can be used

261 in tandem with other methods to examine commission and omission errors. Consider a

262 simple comparison, by intersecting the PPA home range with commonly employed home

263 range techniques MCP and KDE (Figure 4). The PPA home range represents the largest

264 spatial unit such that no omission error occurs, due to explicit consideration of the time

265 geography constraints on animal movement. Potential omission errors are then easily

266 represented as those areas included in the PPA home range, but not in other techniques.

267 Areas not included in the PPA home range but included in other methods can be

268 considered inaccessible regions and an unnecessary source of commission error. With

269 MCP, potential omission errors are likely to occur near edges of MCP home ranges. Due

270 to the convex assumption, MCP home ranges almost always include inaccessible areas as 
271 well (Powell 2000). KDE home range polygons are not guaranteed to even include all

272 sampled telemetry points, therefore explicitly known errors of omission may exist.

273 < approximate location Figure $4>$

274 All measures of home range are indirect and based on specific properties of the

275 telemetry data from which they are derived. Most existing methods use only the spatial

276 properties of telemetry data represented as points. The PPA method provides a

277 complementary view that not only considers spatial information but also temporal

278 information. Using the demonstrated intersection technique, omission errors and

279 inaccessible regions (unnecessary commission error) using existing home range methods

280 can be mapped and quantified. This represents a significant contribution towards home

281 range analysis that carefully considers these types of errors as has been previously

282 suggested (Sanderson 1966). Often studies employ multiple methods when delineating

283 wildlife home ranges to evaluate a range of possibilities (e.g., Righton and Mills 2006).

284 The PPA home range should be included in such studies as it can be used to augment

285 other techniques by providing information on omission and commission errors.

286 In this derivation of PPA home range all geographical space is considered equally

287 navigable. In reality, environmental factors (e.g., topography, land cover, water bodies)

288 influence an animal's ability to traverse the landscape. As well, external factors such as

289 inter- and intra-species competition (Schwartz et al. 2010), and habitat requirements

290 (Sawyer et al. 2007), motivate wildlife movement, and subsequent home range

291 delineations. Optimally, PPA home ranges would be based on the time geography

292 constraints across an unequal surface (see Miller and Bridwell 2009), that considers

293 competition, habitat, topography, and barriers to wildlife movement. Future work should 
294 investigate combining available environmental datasets into animal specific movement

295 cost surfaces. Movement cost surfaces could then be integrated into time geographic

296 analysis to compute more realistic PPA home ranges. However, incorporating movement

297 cost surfaces may take away from the attractiveness of time geography methods due to

298 added complexity.

\section{MANAGEMENT IMPLICATIONS}

300 The concept of home range remains at the core of current research on wildlife movement

301 and habitat analysis, and is frequently adopted as a tool in wildlife management

302 applications. In this article we have presented a new technique for deriving animal home

303 ranges that is simple and intuitive, but also designed specifically for use with emerging

304 temporally dense telemetry datasets, such as those now routinely collected with GPS

305 collars. However, we suggest the PPA approach not be adopted with temporally coarser

306 telemetry data (e.g., VHF collars) as it can lead to overestimation of home range size and

307 misleading interpretations. The PPA home range can be used as a stand-alone measure of

308 animal home range, or to augment existing techniques by identifying potential omission

309 errors and inaccessible areas making it flexible for use with both novel and existing

310 analyses. When performing PPA home range analysis the method for obtaining the $v_{\max }$

311 parameter (e.g., through biological reasoning or by one of the estimation approaches we

312 provide) along with the parameter value should be explicitly stated, as it will influence

313 the resulting home range area. To those wishing to implement the PPA home range

314 technique in their own research we have provided access to a tool for implementing the

315 PPA home range. For more information please go to:

316 http://www.geog.uvic.ca/spar/tools.html. 
16 | Long and Nelson

\section{Acknowledgements}

318 Funding for this work was provided by Canada's Natural Science and Engineering

319 Research Council (NSERC) and GEOIDE through the Government of Canada's

320 Networks of Centres of Excellence program. Thanks to B. Stewart for assistance in

321 programming the implementation tool. The comments and suggestions we received from

322 G. White, N. Lichti, and one anonymous reviewer greatly improved the presentation of

323 this article.

\section{$325 \quad$ Literature Cited}

326 Aebischer, N. J., P. A. Robertson, and R. E. Kenward. 1993. Compositional analysis of 327 habitat use from animal radio-tracking data. Ecology 74:1313-1325.

328 Baer, L. D., and D. R. Butler. 2000. Space-time modeling of grizzly bears. Geographical $329 \quad$ Review 90:206-221.

330 Borger, L., N. Franconi, G. De Michelle, A. Gantz, F. Meschi, A. Manica, S. Lovari, and 331 T. Coulson. 2006. Effects of sampling regime on the mean and variance of home $332 \quad$ range size estimates. Journal of Animal Ecology 75:1393-1405.

333 Burt, W. H. 1943. Territoriality and home range concepts as applied to mammals. Journal 334 of Mammalogy 24:346-352.

335 Cooke, P. 1979. Statistical inference for bounds of random variables. Biometrika 66:367$336 \quad 374$.

337 Dixon, K. R., and J. A. Chapman. 1980. Harmonic mean measure of animal activity 338 areas. Ecology 61:1040-1044. 
17 | Long and Nelson

339 Downs, J. A. 2010. Time-geographic density estimation for moving point objects. Pages

340 16-26 in Proceedings of GIScience 2010, LNCS 6292.

341 Downs, J. A., and M. A. Horner. 2008. Effects of point pattern shape on home-range

342 estimates. Journal of Wildlife Management 72:1813-1818.

343 . 2009. A characteristic-hull based method for home range estimation. Transactions

344 in GIS 13:527-537.

345 Fieberg, J., and C. O. Kochanny. 2005. Quantifying home-range overlap: The importance

346 of the utilization distribution. Journal of Wildlife Management 69:1346-1359.

347 Fieberg, J., J. Matthiopoulos, M. Hebblewhite, M. S. Boyce, and J. L. Frair. 2010.

348 Correlation and studies of habitat selection: problem, red herring or opportunity?

349 Philosophical Transactions of the Royal Society B 365:2233-2244.

350 Frair, J. L., S. E. Nielson, E. H. Merrill, S. R. Lele, M. S. Boyce, R. H. M. Munro, G. B.

351 Stenhouse, and H. L. Beyer. 2004. Removing GPS collar bias in habitat selection

352 studies. Journal of Applied Ecology 41:201-212.

353 Getz, W. M., and C. C. Wilmers. 2004. A local nearest-neighbor convex-hull

354 construction of home ranges and utilization distributions. Ecography 27:489-505.

355 Gurarie, E., R. D. Andrews, and K. L. Laidre. 2009. A novel method for identifying

356 behavioural changes in animal movement data. Ecology Letters 12:195-408.

357 Hagerstrand, T. 1970. What about people in regional science? Papers of the Regional

$358 \quad$ Science Association 24:7-21.

359 Hemson, G., P. Johnson, A. South, R. Kenward, R. Ripley, and D. MacDonald. 2005. Are

360 kernels the mustard? Data from global positioning system (GPS) collars suggests 
18 | Long and Nelson

problems for kernel home-range analyses with least-squares cross-validation. Journal of Animal Ecology 74:455-463.

363 Horne, J. S., E. O. Garton, S. M. Krone, and J. S. Lewis. 2007. Analyzing animal

364 movement using Brownian bridges. Ecology 88:2354-2363.

365 Jennrich, R. I., and F. B. Turner. 1969. Measurement of non-circular home range. Journal

366 of Theoretical Biology 22:227-237.

367 Jonsen, I. D., J. Mills Flemming, and R. A. Myers. 2005. Robust state-space modeling of 368 animal movement data. Ecology 86:2874-2880.

369 Kie, J. G., J. Matthiopoulos, J. Fieberg, R. A. Powell, F. Cagnacci, M. S. Mitchell, J.-M.

370 Gaillard, and P. R. Moorcroft. 2010. The home-range concept: are traditional

371 estimators still relevant with modern telemetry technology? Philosophical

372 Transactions of the Royal Society B 365:2221-2231.

373 Kraak, M. J. 2003. The space-time cube revisited from a geovisualization perspective.

374 Pages 1988-1995 in Proceedings of 21st International Cartographic Conference.

375 Kwan, M. P., and J. Lee. 2004. Geovisualization of human activity patterns using 3D

376 GIS: A time-geographic approach. Pages 48-66 in M. F. Goodchild, and D. G.

377 Janelle, editors. Spatially Integrated Social Science: Examples in Best Practice.

$378 \quad$ Oxford University Press, Oxford.

379 Laver, P. N., and M. J. Kelly. 2008. A critical review of home range studies. Journal of $380 \quad$ Wildlife Management 72:290-298.

381 Miller, H. J. 2005. A measurement theory for time geography. Geographical Analysis $382 \quad 37: 17-45$. 
19 | Long and Nelson

383 Miller, H. J., and S. A. Bridwell. 2009. A field-based theory for time geography. Annals 384 of the Association of American Geographers 99:49-75.

385 Mitchell, M. S., and R. A. Powell. 2008. Estimated home ranges can misrepresent habitat 386 relationships on patchy landscapes. Ecological Modelling 216:409-414.

387 Nielson, S. E., M. S. Boyce, G. B. Stenhouse, and R. H. M. Munro. 2003. Development and testing of phenologically driven grizzly bear habitat models. Ecoscience

Otis, D. L., and G. C. White. 1999. Autocorrelation of location estimates and the analysis of radiotracking data. The Journal of Wildlife Management 63:1039-1044.

392 Parkes, D. N., and N. Thrift. 1975. Timing space and spacing time. Environment and $393 \quad$ Planning A 7:651-670.

394 Powell, R. A. 2000. Animal home ranges and territories and home range estimators. Pages 65-110 in L. Boitani, and T. K. Fuller, editors. Research Techniques in Animal Ecology: Controversies and Consequences. Columbia University Press,

398 Rempel, R. S., A. R. Rodgers, and K. F. Abraham. 1995. Performance of a GPS animal 399 location system under boreal forest canopy. Journal of Wildlife Management 59:543-551.

401 Righton, D., and C. Mills. 2006. Application of GIS to investigate the use of space in 402 coral reef fish: a comparison of territorial behaviour in two Red Sea 403 butterflyfishes. International Journal of Geographical Information Science $404 \quad 20: 215-232$. 
20 | Long and Nelson

405 Robson, D. S., and J. H. Whitlock. 1964. Estimation of a truncation point. Biometrika $406 \quad 51: 33-39$.

407 Sanderson, G. C. 1966. The study of mammal movements: A review. Journal of Wildlife $408 \quad$ Management 30:215-235.

409 Sawyer, H., R. M. Nielson, F. G. Lindzey, L. Keith, J. H. Powell, and A. A. Abraham. 410 2007. Habitat selection of Rocky Mountain Elk in a nonforested environment. $411 \quad$ Journal of Wildlife Management 71:868-874.

412 Schwartz, C. C., C. L. Cain, S. Podruzny, S. Cherry, and L. Frattaroli. 2010. Contrasting 413 activity patterns of sympatric and allopatric black and grizzly bears. Journal of $414 \quad$ Wildlife Management 74:1628-1638.

415 Sharp, N. C. C. 1997. Timed running speed of a cheetah (Acinonyx jubatus). Journal of $416 \quad$ Zoology 241:493-494.

417 Smulders, M. C. A. 2009. Spatial-temporal analysis of grizzly bear habitat use. 418 University of Victoria, Victoria, BC.

419 Swihart, R. K., and N. A. Slade. 1985. Influence of sampling interval on estimates of 420 home-range size. Journal of Wildlife Management 49:1019-1025.

421 Tomkiewicz, S. M., M. R. Fuller, J. G. Kie, and K. K. Bates. 2010. Global positioning 422 system and associated technologies in animal behaviour and ecological research. 423 Philosophical Transactions of the Royal Society B 365:2163-2176.

424 van der Watt, P. 1980. A note on estimation bounds of random variables. Biometrika $425 \quad 67: 712-714$. 
21 | Long and Nelson

426 Wentz, E. A., A. F. Campbell, and R. Houston. 2003. A comparison of two methods to create tracks of moving objects: linear weighted distance and constrained random walk. International Journal of Geographical Information Science 17:623-645.

429 Worton, B. J. 1987. A review of models of home range for animal movement. Ecological $430 \quad$ Modelling 38:277-298.

$431 \ldots$ 1989. Kernel methods for estimating the utilization distribution in home-range

432 studies. Ecology 70:164-168. 


\section{Figure Captions:}

Figure 1: Diagram of Hagerstrand's (1970) time geography. The space-time prism contains the set of all locations accessible to an individual given telemetry fixes at $t_{1}$ and $t_{2}$, and a velocity parameter $\left(v_{\max }\right)$. The projection of the space-time prism onto the geographical plane is called the potential path area (PPA), used here for delineating wildlife home ranges.

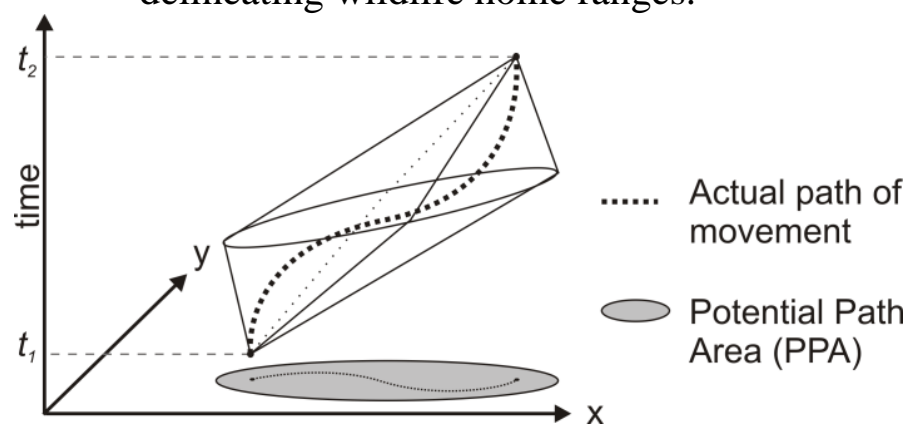


Figure 2: a) Pins-and-strings method for generating PPA ellipses. The length of the string is equal to the longest distance the animal could travel $\left(D_{\max }\right)$ given parameter $v_{\max }$ and the time difference between points. $\left.\mathrm{b}\right)$ Geometric properties of a PPA ellipse with telemetry points $i$ and $j$. $C P$ is the center point and $d$ is the Euclidean distance between points $i$ and $j ; a$ and $b$ are lengths of the major and minor axis respectively; and $R_{\theta}$ is the rotation angle. c) Computation of the PPA home range involves combining multiple $(n-1)$ PPA ellipses.

a)

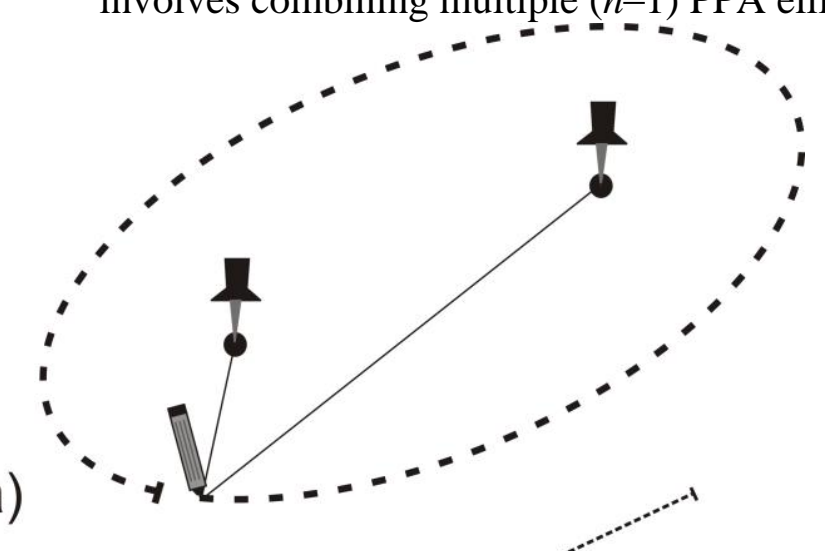

b)

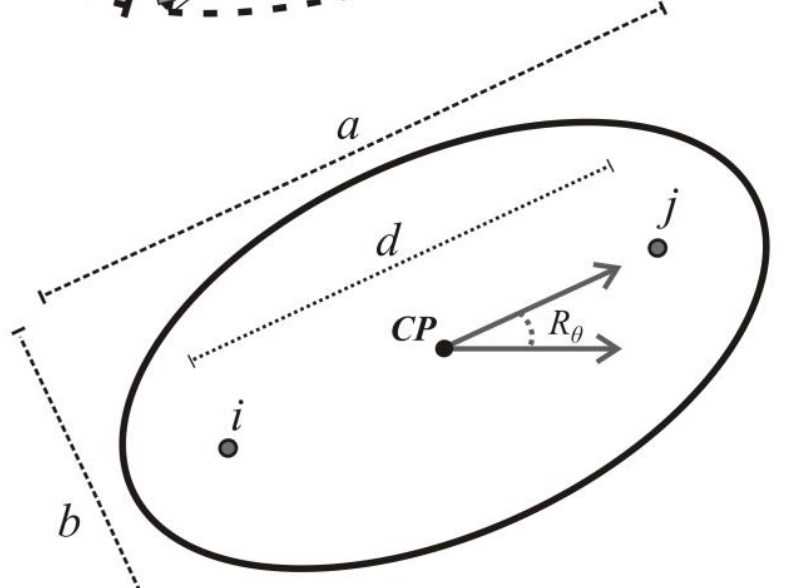

C)

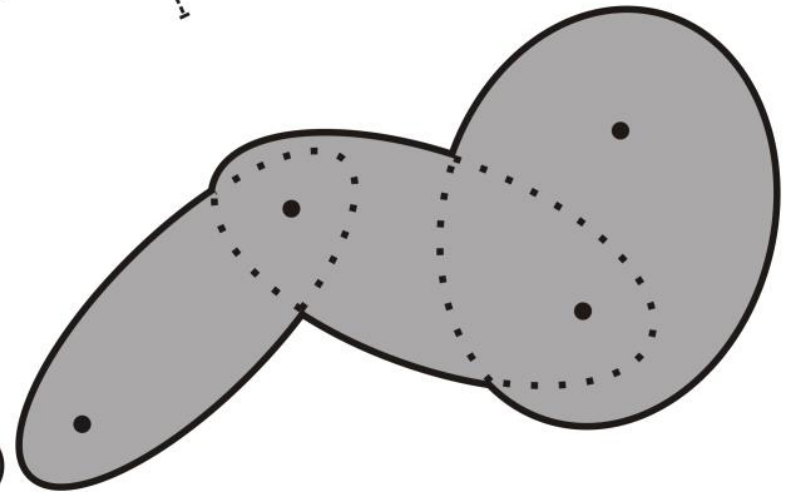


Figure 3: Home range polygons for a simulated dataset with $n=2000$ (top) re-sampled to $n=500$ (bottom) using MCP (a \& d), KDE (b \& e) and PPA (c \& f).
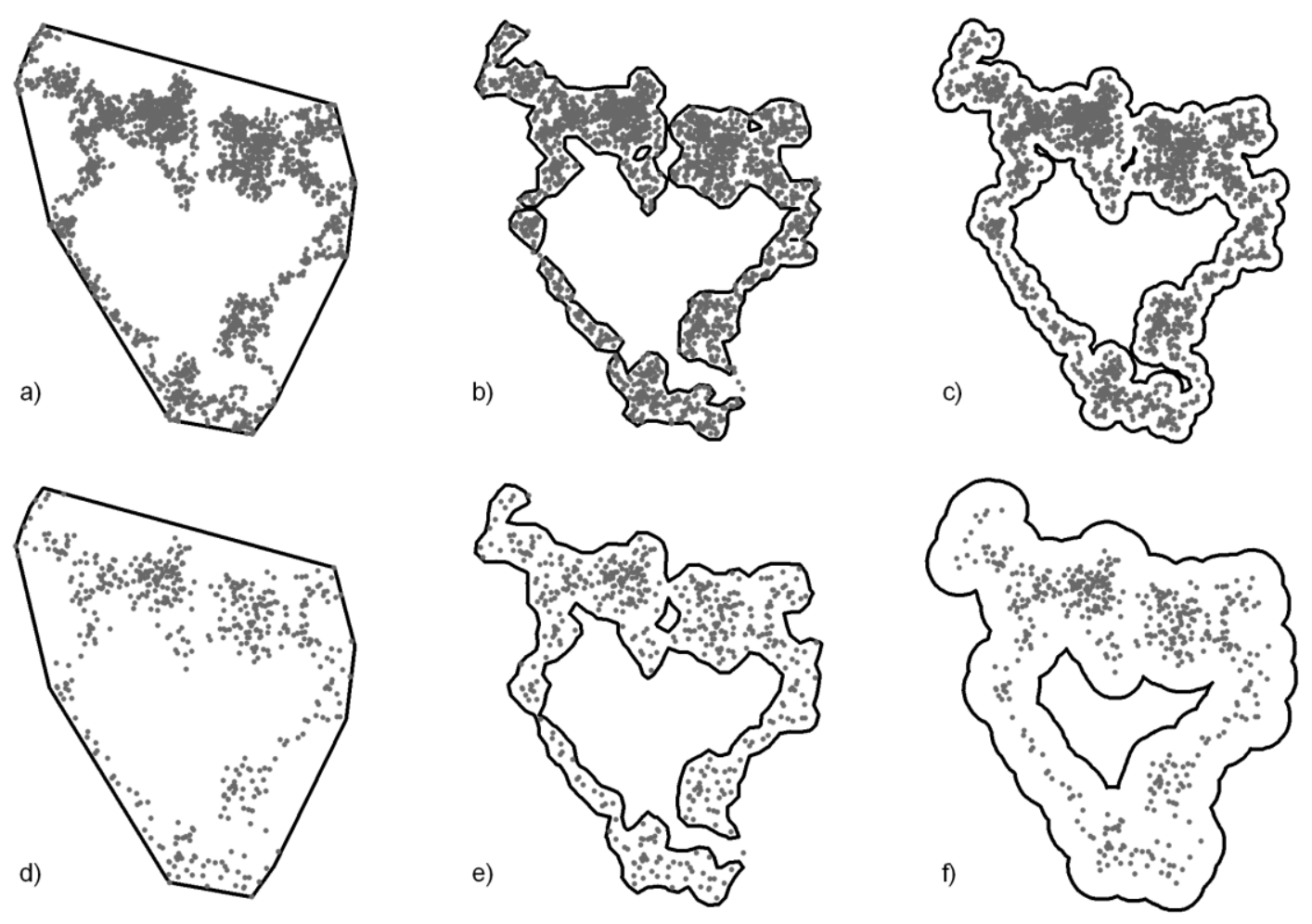
Figure 4: Intersections between a) MCP \& PPA and b) KDE \& PPA (for $n=2000$ ); demonstrating how PPA home ranges can be used to augment existing techniques by identifying omission errors and inaccessible areas.
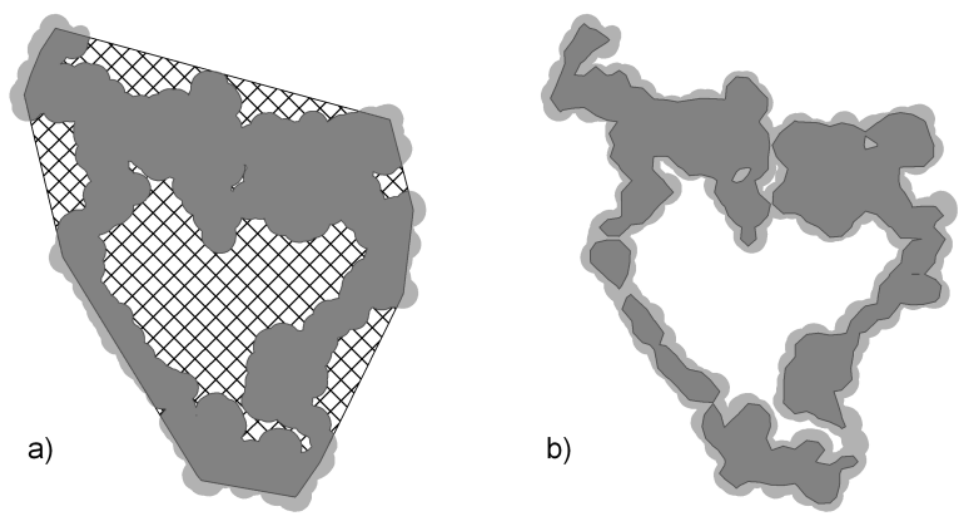

\section{Area of intersect}

Potential omission error Inaccessible area (extra commission error) 
26 | Long and Nelson 